\title{
Medica! Hypotheses
}

\section{Riley-Day Syndrome, Brain Stimulation and the Genetic Engineering of a World Without Pain}

\author{
L. S. MANCINI \\ 25 Nottingham Terrace, Buffalo, New York 14216, USA
}

Abstract - Riley-Day Syndrome, a genetic disorder in which there is impaired ability or inability to feel pain, hot and cold, is cited as an example of evidence that the commonplace notion that life cannot be painless is not necessarily valid. A hypothesis is presented to the effect that everything adaptive which is achievable with a mind capable of experiencing varying degrees of both pleasure and pain (the human condition as we know it) could be achieved with a mind capable of experiencing only varying degrees of pleasure. Two possible approaches whereby the human mind could be rendered painless are a schematically-outlined genetic approach, which would or will probably take thousands of years to implement, and a brain stimulation approach that could be effected by means of a noninvasive, contactless, transcranial, deep-neuroanatomic-site-focusable, electromagnetic and/or ultrasonic (and/or, conceivably, other kind of) brain pacemaker which could be developed within a few years. In order to expedite the relief of all kinds of suffering and the improvement of the human condition in general, it is advocated that prompt and concerted research effort be directed toward the development of such a brain pacemaker.

\section{Introduction}

In this article the concept of pain should be construed in its broadest sense. It should be thought of not merely as bodily suffering but rather as any unpleasant or distressing experience, whether it be of bodily pain, nausea, dyspnca, pruritus, hunger, thirst, fear (anxiety), depression, anger, etc. Most people seem to regard pain as a necessary evil of life. Such rationalizations as 'no pain, no gain', which are widely invoked, may he adaptive in terms of helping one to deal with life's current reality, but this does not mean they are or will prove to be insurmountably valid for all time. On the contrary, one will find that the people who are the most productive and efficient at their work tend to be the ones who enjoy it the most.

Or people will ask, 'How would you be able to recognize pleasure if you never experienced pain?' On the contrary, one does not need to have been tortured in order to enjoy fine music or fine dining. Or people will contend that hap- 
piness is a matter of free will. In effect, those who are happy are so because they choose and work to be. Those who are unhappy are so because they do not choose or work to be happy. However, in view of the now well-documented biological and, in some cases, hereditary bases of many of the depressive, anxiety, and psychotic disorders, to say nothing of adverse external circumstances such as the occurrence of natural disasters, over which one cannot possibly (except, conceivably, superstitiously) have any control, any self-deterministic theory of happiness level seems seriously flawed or altogether incredible.

Or people will rationalize: 'If you were happy all the time, then you would be bored'. On the contrary, this statement makes no sense, because happiness and boredom are mutually exclusive states. If one were to consider only the happiest (the most enthusiastic and contented) one or two percent of the human population, it would be surprising if one would find that these people spend even a small fraction of their time being bored. Hence, even given the existing state of affairs, it is not necessarily humanly impossible to be happy most of the time. Some electrophysiological ways in which pleasure and pain could be measured and quantified are suggested below.

\section{Riley-Day Syndrome}

A rare genetic disorder known as Riley-Day Syndrome or familial dysautonomia, i.e. FD (1, $2,3,4)$, which has an autosomal recessive means of transmission, was first identified in 1949. It occurs almost exclusively in descendants of the Eastern European Ashkenazy branch of Judaism, although it has been diagnosed occasionally in members of other religious, ethnic, and/or racial groups.

The syndrome is evident from the time of birth in terms of difficulty in feeding, episodes of unexplained fever and pneumonia, and failure to thrive. Its symptoms and signs as a life-long disorder include defective lacrimation with an inability to shed tears when crying, corneal ulceration, absent corneal, axonal, and tendon reflexes, unstable blood pressure with episodes of hypertension and postural hypotension, unstable body temperature, vomiting spasms, profuse sweating, sialorrhea, impairment of vestibular function, repeated infections, an initial delay in mental development (with the subsequent achievement of intellectual parity with one's peers by age 4), difficulty or inability to suck or chew with impaired pharyngeal and esophageal motility, esophageal and intestinal dilation, absence of taste buds or fungiform papillae with inability to taste food, a marked tendency to develop symmetric blotchy erthematous skin rashes, especially in connection with eating or emotional stress, emotional lability with a nervous system which is unstable in the sense that strong emotions, whether pleasing or distressing, frequently lead to episodes of loss of consciousness, stunted growth and, most significantly from the standpoint of this article, an impaired ability or inability to feel pain, and an impaired ability or inability to feel (or distinguish between) hot and cold, although there is relative preservation of pressure and tactile sense.

The syndrome is considered to represent a disturbance of both sensory and autonomic functions, both parasympathetic and sympathetic. Compared to individuals who are not afflicted with the syndrome (i.e., neurologically normal individuals), there is a diminution in the number of sympathetic and parasympathetic ganglion cells and, to a lesser degree, in the number of nerve cells in the sensory ganglia. There is a paucity of small myelinated and unmyelinated nerve fibers, which explains the impairment of tempcrature and pain sensation. There is increased excretion of homovanillic acid and decreased excretion of vanillylmandelic acid and methoxyhydroxyphenylglycol. There is also an abnormally low concentration of serum dopamine beta-hydroxylase, the enzyme that converts dopamine to norepinephrine.

There are about 300 known cases in the United States, but the true incidence, which would amount to a larger number, among American Jews, is estimated to lie between 1 in 10000 and 1 in 20000 with a carrier frequency of 1 in 50 to 1 in 70 . According to Bundey and Brett (1), $25 \%$ of afflicted children are dead by age 10 and $50 \%$ by age 20 , usually as a result of pulmonary problems secondary to bronchial hypersecretion or inhalation of stomach contents during attacks of vomiting. Apparently due to consciousnessraising brought about by the New York based Dysautonomia Foundation and the concerted efforts of a pediatrician, Dr. Felicia Axelrod (2), who has centered her life's work on FD, improved supportive and symptomatic treatment has resulted in more afflicted children surviving to adulthood. Nonetheless, so far no one known 
to have this condition has survived beyond the fifth decade of life. There is, as yet, no definitive treatment.

Although FD probably originated well before its date of initial identification (1949), it is at least ironic that a genetic condition involving an inability to experience bodily pain would emerge primarily among a group of people who had been subjected, unfortunately and unpardonably (over a period of centuries or millennia), to extraordinary amounts of pain (which culminated) only a few years earlier, during the heinous period of Nazi domination connected with World War II $(2,3,5)$.

\section{The underlying implications of Riley-Day Syndrome}

Although from a superficial standpoint the existence of FD as a disease entity may seem to underscore the indispensability of pain to the processes whereby an organism adapts to its environment, closer scrutiny will reveal that this indispensability may be more illusory and circumstantial than it is real and immutable. The most important point to be deduced from FD is that life without a phenomenon which most of us assume is an unavoidable part of life (that is, the capability of experiencing bodily pain) is not only conceivable but actually occurs in some cases. If FD did not exist, it would be easier for anyone to claim that life without the capability of experiencing bodily pain is an impossibility.

If the genetic modification which underlies FD can result in a life without bodily pain, then it is quite conceivable that other genetic modifications could result in lives devoid of the capabilities of experiencing any and all other forms of pain, including everything from nausea to frustration and hostility (variants or subtypes of anger).

In fact, there is another condition, even rarer than FD, known as congenital indifference to pain (1), in which the individual does recognize a painful stimulus as such but perceives it as no more distressing than a touch or a ticklc, that is, not distressing at all. These patients can learn to take precautions that will minimize the possibility of trauma. So far, the nervous systems of such individuals have not been shown to be abnormal in any way (1). Nonetheless, this condition of lifelong unreactivity to pain does appear to adhere to a genetic pattern of inheritance, in some cases autosomal dominant, in others autosomal recessive. Because it appears to be possible to compensate adaptively for this condition, it would appear to be a better candidate for genetic modeling for a painless world than FD, but it too leaves much to be desired, both because it tends to be maladaptive and because it does not preclude forms of pain other than the distress of bodily pain.

\section{How life could be painless without being maladaptive}

Let us now broach the question of how, in psychodynamic terms, we could go through life as adaptively (or more so, and ccrtainly more enjoyably) without the capability of experiencing any kind of pain as we do in our current condition of being equipped with that capability. For example, if we did not have anxiety - specifically, the fear of getting killed - what would prevent us from driving our cars into the oncoming traffic? An answer is as follows. We would not want to genetically engineer ourselves so that we would be rid of the anxious impulses that deter our driving into traffic without putting anything adaptive in their place. However, what could adaptively be put in place of these anxious impulses would be pleasure-diminishing impulses with or without the added adaptive benefit of reflexly avoidant impulses. Hence, rather than producing pain (anxiety), the thought or anticipation of the possibility of driving into oncoming traffic would produce a marked diminution of the high level of baseline pleasure, with or without an accompanying unconsciously motivated reflexive avoidance response, which would prevent the injurious behavior just as reliably as the anxiety currently does. The option of having unconscious reflex avoidance associated with any perception of potential danger, while not strictly necessary in terms of protective motivational wherewithal, might facilitate faster reaction times (because less interneuronal processing would be entailed) than would pleasure diminution alone associated with any perception of danger.

Furthermore, a proportional factor could be built into the system (the genetically engineered mind) so that the more dangerous the situation (for example, the closer one's actual position to the oncoming traffic), the more marked the diminution of pleasurable impulses (and, possibly, the stronger the unconsciously-motivated avoidance responses) would be.

By generalizing from this example, it is 
possible to appreciate that everything adaptive which can be achieved with a mind capable of experiencing varying degrees of both pleasure and pain (the current human condition) could be achieved with a mind capable of experiencing only varying degrees of pleasure or a mind capable of experiencing such pleasure simultaneously with varying intensities of unconsciously mediated avoidance behavior. The possibility of leading a strictly painless yet adaptive life would constitute an improved human condition.

This improved condition could be achieved in either one of two different ways, with each of them having its respective pros and cons. One way in which it could possibly be achieved would be with a contactless, noninvasive, transcranial brain pacemaker which could also be called a noninvasive neuroprosthesis or brain stimulator. The essence of the method whereby brain pacemaking or brain stimulation could be used to effect the improved human condition is delineated in another paper (6) and, therefore, need not be repeated here.

Brain stimulation experiments done on animals confirm the widely acknowledged point that 'stress kills' which is to say that the unpleasant aspect of stress or distress affects organisms adversely not only in terms of the quality of life, but also in terms of the duration of life. Prolonged unconditional, unavoidable stimulation, lasting 24 hours or more, of areas within an animal's brain which cause the animal to show all of the signs of extreme distress (i.e., 'punishment centers', areas to which an animal will promptly terminate stimulation if given the means to do so) has been observed to actually cause the animal to become severely ill and die (7). Perhaps needless to say, such experiments are horrendously cruel and, while carrying out such experiments even once is unconscionable regardless of the extent to which scientific knowledge might be augmented thereby, certainly should never be done again.

\section{How genetic engineering could be used to effect a painless improved human condition}

There is currently a good deal of speculation and controversy regarding the prospect of mapping and sequencing the entire human genome. Dr. Leroy Hood, a pioneer in the field of biotechnology (8), estimates that it will take us at least hundreds of years to decipher the multitude of messages contained in the human genome.'

We know that some people have a low threshold of pain and other people have a high threshold of pain. And this would seem to be true regardless of what kind of pain we are talking about. It is also quite likely, as is exemplified by $F D$, that there is a strong genetic basis for the height of a person's threshold for any kind of pain. Let us consider two different kinds of people. The first kind of people become greatly distressed when confronted with adverse circumstances and only tolerably placated when confronted with favorable circumstances. Let us describe these people as pain-dominated (PAD). The other kind of people become only mildly displeased when confronted with adverse circumstances and become greatly elated when confronted with favorable circumstances. Let us describe these people as pleasure-dominated (PLD).

The majority of most present-day populations would fall somewhere in between the two extremes of being PAD and PLD. However, let us suppose that for any given person there is some electrophysiologically discernible characteristic (of the computerized-spectral-analyzed electroencephalogram or EEG, magnetoencephalogram or MEG, electromyogram or EMG, or whatever) which can be detected whenever the person is experiencing any kind of pleasure. This characteristic can be referred to as the pleasure characteristic (PLC). Because the mind gives the same 'pleasurable' label to all different kinds of pleasure (in other words, the mind is aware of both the differences and the fundamental similarity among the various different kinds of pleasure), it makes sense that there would be some potentially electrophysiologicallydetectable, common denominator of all of them. Similarly, suppose that for any given person there is some electrophysiologically discernible characteristic which can be detected whenever the person is experiencing any kind of pain. This can be called the pain characteristic (PAC).

Then, by measuring any person's amplitudes, duration, and frequency of occurrence of PLC and PAC, one could determine whether the person is more PLD or more PAD. If we were to measure and record the PLC and PAC values for every person in a population over a period of time, we would then be able to identify both those who are extremely PLD and those who are extremely PAD. Suppose the $1 \%$ of the popu- 
lation who are most PLD (1\%-PLD) and the $1 \%$ who are most PAD (1\%-PAD) have been isolated. One would probably then be able to verify that the $1 \%$-PLD and the $1 \%$-PAD groups, respectively, (approximately, at least after individual differences in stress exposure have been taken into account) constitute or at least correlate positively with the $1 \%$ of the population with the highest and lowest pain thresholds. Using some arbitrary scale of pain threshold, suppose the average pain threshold value of the $1 \%$-PLD group is 100 and the corresponding value for the $1 \%-\mathrm{PAD}$ group is 10 . After the task of having sequenced the entire human genome has been completed, suppose we move on to the task of sequencing each person's entire genome or, at least, for the purpose in question here, the task of sequencing the genome of each person in either of these two $1 \%$ subpopulations.

One should then look for systematic differences between the DNA sequences of the $1 \%$-PLD group and the 1\%-PAD group. The likelihood seems high that there would be at least one common denominator, or possibly a number of different patterns of common denominator(s), of DNA sequencing among the 1\%-PLD group (PLD-DNA) and at least one common denominator (different from PLD-DNA) of DNA sequencing among the 1\%-PAD group (PAD-DNA). Then, by noting the trend of differences between the DNA-sequence common denominator patterns for the pleasure-dominated group and the DNA-sequence common denominator patterns for the pain-dominated group, one could possibly extrapolate a DNA sequence common denominator that would characterize people with an ultra-high pain threshold of, for example, 1000 . Let us refer to this extrapolated sequence as a super-plcasurcdominated DNA or super PLD-DNA sequence. Anyone with such a DNA sequence in her or his genome would have a very high (possibly higher than any level or value which nature, unassisted, has bestowed upon anyone) threshold of pain, perhaps comparably as high for all kinds of pain as the thresholds for mechanical and thermal pain of the prototypical FD sufferer, which could mean unreachably high for all kinds of pain.

Any individual with the extrapolated DNA sequence in his or her genome might actually still be mildly pleased (or have a neutral affect) when confronted with adverse circumstances and would undoubtedly be joyously elated when confronted with favorable circumstances. Such an individual could never experience any pain. Her/his mood could only vary between neutral affect when confronted with catastrophic circumstances (but the pleasure-seeking nature of all organisms, rcgardless of height of pain threshold, would still motivate such a person to do whatever possible to calmly undo or compensate for catastrophic circumstances), and joyous elation when confronted with even the ordinary homeostasisconducive circumstances, such as the pervasiveness of ample amounts of oxygen for the purpose of breathing, which most of us take for granted.

Then, one could implement in vitro genetic engineering by microinjecting cvcry fcrtilized human egg with the recombinant super PLDDNA sequence(s) that would replace the existing homologous (mediocre) PLD-DNA and/or PADDNA sequence(s) so that all humans born after the perfection of the recombinant super PLDDNA sequence microinjection technique would have a pain threshold of approximately 1000 and be unable to experience pain of any kind.

Or, when and if in vivo genetic transformation techniques are ever perfected, whereby every cell in the body of a living organism at any stage in its life span can simultaneously undergo homologous insertion of any desired recombinant DNA sequence encoding for any desired gene(s) coupled with the delction of the homologous less desirable DNA sequence encoding for any less desirable gene(s), then all individuals alive at the time of the achievement of such technical capability, regardless of their age at that time, could be converted from being PAD or PLD typc people to being super-pleasure-dominated people incapable of experiencing pain of any kind.

But what if these elation-prone, superpleasure-dominated genetic transformers with unreachably high pain thresholds prove to be aimlessly and recklessly euphoric, like many present-day psychiatric patients who are afflicted with mania, as in manic-depressive illness? Such individuals would have little or no inclination toward concerted, constructive, and productive activities (i.e., learning and working; work that is of value to society), and a marked inclination toward consumptive or libidinal activities such as wild spending sprees, gambling, alcohol abuse, sexual indiscretion, overcating, etc. Even though they would be incapable of suffering, they would be relegated to relatively unfulfilling, hence dull, minimally pleasurable lives, because nothing and no one would reward them for their unproductivity. Their lives would be devoid of a sense of 
purpose which is essential to happiness. They would be a burden to society and to themselves. Their problems could be solved by implementing methods whereby their vast potential pleasure could be actualized and harnessed or channeled into constructive, productive, adaptive activities and pursuits.

One way of doing this would be by implementing the brain stimulation paradigms delineated in another paper (6), whereby learning and working could be made at least as pleasurable and probably (by dint of the intrinsic pleasurableness of diversification of knowledge and skills) more pleasurable than consumptive or libidinal activities.

Another way, a genetic way, of accomplishing this would be by looking for and isolating common denominators of DNA sequencing that characteristically are present within the genomes of individuals (regardless of whether they are PLD or PAD) who do function productively and adaptively. And then, by in vivo insertion of the productivity-/adaptiveness-characteristic DNA sequences into every cell in the bodies of these unproductive, maladaptive super PLD individuals, which would effect homologous replacement of DNA sequences encoding for unproductive, maladaptive tendencies with DNA sequences encoding for productive, adaptive tendencies, they could convert themselves from pleasureless, painless, unproductive members of society into elated, productive members thereof. Hence, the goal of adaptive, painless, and pleasurable living could be achieved genetically as well as by brain stimulation.

\section{The pros and cons of the two different approaches}

The genetic engineering approach to achieving painless living would be the definitive one, far superior to brain pacemaking, primarily because, with the former, the individual would not have to be dependent upon or encumbered with external gadgetry or subject to conceivable side effects of long-term brain stimulation. However, the drawback of the genetic approach is that it may take a very long time, perhaps thousands of years, to implement. Such amounts of time would certainly make sense on the time scale of Dr. Hood. In sharp contrast to this greatly extended time scale, noninvasive, contactless brain stimulation or pacemaking, which could be used to accomplish essentially the same goal, that of painless yet adaptive living, could be developed within a few years. Dr. Robert G. Hcath, a pioneer in the use of surgically implanted electrodes to effect neuropsychiatrically relevant brain stimulation, has indicated that an ultrasound-emitting device could be built (ostensibly as early as any time between the present moment and the early part of the 21st century) which could activate the brain's 'pleasure centers' without having to go inside the skull. And, in line with his claim is a prediction that, by the year 2005 , family physicians will be using such a device on a routine therapeutic basis (9).

All of the technological ingredients that would have to be brought together in order to construct such a device already appear to exist. The combined use of electromagnetism and ultrasound (as opposed to ultrasound alone), as suggested by W. J. Fry (10) and affirmed by his brother, F. J. Fry (11), might more readily facilitate the goal of developing a contactless, noninvasive brain pacemaker capable of exciting or suppressing any small or large area(s) in the living human brain. The principal drawbacks of brain pacemaking would be that it would entail the possibility of periodic equipment failure or malfunctioning, the burden (even if a very small, light-weight one) of having to carry around a pacemaker wherever one wanted to go while still having the benefit of its use, and the possibility of some as yet undetermined side-effect(s) which, according to the observations of Barker et al (12), are unlikely to prove prohibitive.

Genetic engineering could enable each person to be whatever s(he) wants whenever s(he) wants

Dr. Hood also predicts (13) that 'It isn't that [through genetic engineering] we'll be able to design individuals whose intelligence is increased by a factor of three. It isn't that we'll be able to change physical attractiveness or emotional stability.' Given the virtually universal human motive toward self-improvement, it appears doubtful that this prediction will prevail through the extent of time. Let us not think in the authoritarian terms of some individuals genetically engineering the characteristics of others. Instead, let us think in the egalitarian terms of each individual genetically re-engineering herself/himself according as $s($ he) pleases. What is being suggested here is that in the distant future, 
by means of in vivo genetic transformation techniques effected with recombinant DNA or some other biotechnological tool(s), it will be possible for any person (or other kind of organism) to be an introverted, academically-oriented, purplehaired, orange-eyed, 10 foot tall white male with an IQ of 160 on any given day and a party-going, humorous, green-haired, green-eyed, three foot tall green female with an IQ of 200 on the next day. Stated in more general terms, it will become possible for each one of us (that is, anyone alive during the future era in question) to be whatever we want to be whenever we want to be. Some of us may choose to take a DNA pill that will cause us to sprout a pair of wings whenever an automobile is not readily available to us. Granted: all of this may be thousands of years away, but compared to the eternity that stretches ahead, the amount of time in question is minuscule.

Some may object that if each of us were able to change our mental and/or physical characteristics at any given time, then the notion of individual identity would be essentially lost. Undoubtedly, the objection is at least partly valid (however, the philosophical implications will not be delved into here), but the advantages of such a greatly augmented arena of potential endeavor would seem to greatly outweigh the disadvantages. In a world equipped with virtually instantaneous, or at least high-speed, selfdetermined in vivo genetic transformation, no one would ever have any reason to feel inferior to or less fortunate than anyone else, because whatever characteristic(s) one might envy in another person, one could incorporate into one's own being almost as quickly as the envious impulses could emerge. Hence, the ideal of all humans being equal could be realized in terms of each individual having the same (infinitely variable) genetic potential. If this were the case, both egotism and the competitive spirit would become extinct, but their disappearance from the world really would not be a substantial loss; in fact, it would be a gain for everyone.

\section{Conclusion}

In the meantime, that is, the intervening thousands of years between now and the successful implementation of genetic engineering techniques which might create the possibility of painless living, it would be advisable to develop a brain pacemaker and then to determine whether or not its drawbacks could be eliminated or minimized to a tolerable level. Moreover, as one of its virtually limitless potential therapeutic applications, it is conceivable that such a pacemaker, by suppressing maladaptive neuronal impulses (such as those underlying the vomiting attacks) and by exciting adaptive impulses (such as those that could underlie better coordinated pharyngeal and esophageal motility during eating), could provide more effective therapy for Riley-Day Syndrome than any that is currently available by minimizing or eliminating such problems as the aspiration of vomitus, impaired eating ability, etc.

\section{References}

1. Bundey S, Brett E M. Genetics and Neurology, p 206. Churchill Livingstone, Edinburgh, 1985.

2. Axelrod FB, Sein ME. Caring for the Child with Familial Dysautonomia - a Treatment Manual. Dysautonomia Foundation Inc., New York, 1982.

3. Adams R D, Victor M. Principles of Neurology. 3rd edition, p 991. McGraw-Hill, New York, 1985.

4. Axelrod F B. Report on the Dysautonomia Research Symposium. Dysautonomia Foundation Inc., New York, May, 1987.

5. Chusid J G. Correlative Neuroanatomy and Functional Neurology. 16th edition, p 148. Lange Medical Publications, Los Altos, California, 1976.

6. Mancini L. Brain stimulation to treat mental illness and enhance human learning, creativity, performance, altruism, and defenses against suffering. Medical Hypotheses 21: 209-219, 1986.

7. Guyton A C. Textbook of Medical Physiology. 7th edition, p 680. W. B. Saunders Company, Philadelphia. 1986.

8. Hood L. Biotechnology and medicine of the future. The Journal of the American Medical Association, March 25: $1837-1844,1988$.

9. Clarke A C. July 20, 2019, Life in the 21st Century, p 223. Macmillan Publishing Company, New York, 1986.

10. Fry W J. Electrical stimulation of brain localized without probes - theoretical analysis of a proposed method. The Journal of the Acoustical Society of America 44, 4: 919 931. 1968.

11. Fry F J, personal communication, May 27, 1987.

12. Barker A T, Freeston I L, Jalinous R, Jarratt J A. Magnetic stimulation of the human brain and peripheral nervous system: an introduction and the results of an initial clinical evaluation. Neurosurgery 20, 1: 100-109, 1987.

13. Davis J. Leroy Hood: automated genetic profiles. Omni, November: 116-156, 1987. 